\title{
Laparoscopic Small Bowel Length Measurement in Bariatric Surgery Using a Hand-Over-Hand Technique with Marked Graspers: an Ex Vivo Experiment
}

\author{
Nienke Slagter ${ }^{1,2}$ ( . Mette van Wilsum ${ }^{1}$ - Loek J. M. de Heide ${ }^{1}$ Ewoud H. Jutte ${ }^{1} \cdot$ Mirjam A. Kaijser ${ }^{1}$. \\ Stefan L. Damen ${ }^{1}$. André P. van Beek ${ }^{3}$ - Marloes Emous ${ }^{1}$
}

Received: 25 November 2021 / Revised: 2 January 2022 / Accepted: 14 January 2022 / Published online: 24 February 2022

(c) The Author(s) 2022

\begin{abstract}
Introduction Tailoring limb length in bariatric surgery is a subject of many studies. To acquire the optimal limb length, accurate measurement of the small bowel length is essential.

Objective To assess the intra- and inter-individual variability of laparoscopic bowel length measurement using a hand-overhand technique with marked graspers.

Method Four bariatric surgeons and four surgical residents performed measurements on cadaver porcine intestine in a laparoscopic box using marked graspers. Each participant performed 10 times a measurement of three different lengths: 150, 180 , and $210 \mathrm{~cm}$. Acceptable percentage deviation from the goal lengths was defined as less than $10 \%$, while unacceptable deviations were defined as more than $15 \%$.

Results The bariatric surgeons measured the 150-, 180-, and 210-cm tasks with $4 \%(C I 0.4,9),-6 \%(C I-11,-0.8)$, and $1 \%(C I-4,6)$ deviation, respectively. In total, the bariatric surgeons estimated 58 out of 119 times $(49 \%)$ between the margins of $10 \%$ deviation and 36 times (30\%) outside the $15 \%$ margin. Considerable inter-individual differences were found between the surgeons. The surgical residents underestimated the tasks with $12 \%(C I-18,-6), 16 \%(C I-19,-13)$, and $18 \%$ $(C I-22,-13)$, respectively.

Conclusion Bariatric surgeons estimated bowel length with on average less than $10 \%$ deviation. However, this still resulted in $30 \%$ of the measurements with more than $15 \%$ deviation. There were considerable inter-individual differences between the surgeons and residents structurally underestimated the bowel length. Ascertainment of measurement accuracy and adequate training is essential for bariatric procedures in which limb length is of importance.
\end{abstract}

Keywords Bariatric surgery $\cdot$ Limb length $\cdot$ Small bowel length $\cdot$ Laparoscopic bowel length measurement

\section{Introduction}

\section{Key Points}

- Bariatric surgeons estimated bowel length with on average less than $10 \%$ deviation.

- This still resulted in $30 \%$ of the estimated lengths with more than $15 \%$ deviation.

- There were inter-individual differences between the bariatric surgeons.

- Surgical residents structurally underestimated the length of the small bowel.

\section{Nienke Slagter}

N.Slagter@umcg.nl; nienke.slagter@mcl.nl

Extended author information available on the last page of the article
The Roux-en-Y gastric bypass (RYGB), the one anastomosis gastric bypass (OAGB), and single anastomosis duodenoileal bypass with sleeve gastrectomy (SADI-S) are effective and often performed bariatric procedures with comparable outcomes of weight loss and nutritional deficiencies [1-3]. The optimal lengths of the alimentary limb and biliopancreatic (BP) limb remain a topic of discussion. The aim of an optimal limb length is to achieve ideal results in terms of weight loss while minimizing the chance of nutritional deficiencies [4-6]. In RYGB, a longer alimentary limb seems to have no influence on weight loss outcomes, whereas a longer BP-limb results in more weight loss overall [6-8]. 
In the discussion about the optimal limb length, the accuracy and precision of limb length measurement are essential. Inadequate measurement results in over- or under-estimation of a bowel segment. This may have clinical consequences in terms of weight loss, rates of malnutrition, and vitamin deficiencies. In gastric bypass surgery different techniques are performed to measure the small bowel length [9]. Some surgeons use a rope or ruler to measure the segments of the small bowel [10]. Others use graspers or other laparoscopic instruments as a reference tool to stepwise estimate the length of the small bowel [11]. Regardless of the performed measurement method, laparoscopic small bowel measurement remains challenging due to the influence of limited range of motion, the high flexibility of the bowel structure, and the two-dimensional imaging of a three-dimensional bowel [12]. There is limited literature investigating the accuracy of laparoscopic small bowel measurement performed in bariatric surgery. The aim of this study was to assess the intra- and the inter-individual variability of laparoscopic small bowel length measurement using a hand-over-hand technique with marked graspers in an ex vivo experiment.

\section{Method}

\section{Participants}

This is a single-center ex vivo experiment including all four bariatric surgeons and four surgical residents of a nonacademic teaching hospital in the northern Netherlands. Baseline data were collected of all participants including, age, gender, laparoscopic experience, and bariatric experience. For cadaver studies, no ethical approval is required in the Netherlands. The intestine of the porcine cadavers was supplied by a registered slaughterhouse and the use of animal by-products was approved by the Netherlands Food and Consumer Product Safety Authority.

\section{Laparoscopic Simulation}

A laparoscopic box training system (Lapstar model 2, Camtronics B.V. Son, Netherlands) with a fixed camera was used to simulate the laparoscopic abdominal surgery. The total small bowel of a porcine cadaver was placed in the laparoscopic box (Fig. 1). Participants were instructed to perform the stepwise hand-over-hand technique using two metallic graspers to pass the small bowel in estimated steps of $5 \mathrm{~cm}$ (video 1). The length of the antimesenteric border is measured with the bowel in normal position and without applying traction. In our bariatric center, the hand-over-hand measurement technique is the laparoscopic measurement method performed in daily practice. Comparable with the used graspers in bariatric surgery, one of the instruments had a $4.5-\mathrm{cm}$ marker as a reference point.

\section{Laparoscopic Bowel Measurement}

Each participant completed three rounds of 10 measurements on different days, estimating randomly assigned lengths of 150,180 , and $210 \mathrm{~cm}$. The start and endpoints of the measurements were marked with color-coded sutures. Two observers, blinded to the assigned tasks, each measured the actual length of the marked segments outside the laparoscopic box trailer using a tape measure. These measurements were performed immediately after the laparoscopic measurements were completed, to minimize the time difference between the laparoscopic and tape measurements. The bowel was measured with the same technique as the laparoscopic measurements: at the antimesenteric border with the bowel in normal position and without applying traction. The mean of the two tape measure lengths was used as actual measured length. The participants did not receive any feedback on their results until after the experiment to eliminate any influence of learning.

\section{Statistical Analysis}

The individual scores of the participants were presented as mean \pm standard deviation. The percentage deviation from the goal lengths were analyzed by a mixed model for repeated measures analyses, correcting for the dependency of the repeated measurements. The percentage deviation measured by the bariatric surgeons was compared to the surgical residents. Individual percentage deviation of the bariatric surgeons was compared for each measured task. Acceptable variation in small bowel length measurement was defined as less than $10 \%$ deviation from the actual bowel length. Unacceptable variation was defined as more than 15\% deviation from the actual bowel length (Fig. 2). Statistical analyses were assessed in SAS/STAT $®$ software.

\section{Results}

One measured value was excluded due to an incorrect measurement. Three of the bariatric surgeons have performed more than 2000 laparoscopic bariatric surgical procedures during their career and one of the bariatric surgeons performed more than 500 bariatric surgical procedures (Table 5, appendix). As shown in Table 1, the mean measured lengths of the $150-\mathrm{cm}$ task by the four bariatric surgeons individually were $131 \pm 25,158 \pm 17,156 \pm 19$, and $158 \pm 10 \mathrm{~cm}$, respectively. As a group, the surgeons estimated 22 out of 39 times (56\%) between the lower 
and upper margins of $10 \%$ deviation and 7 times (18\%) outside the $15 \%$ margin. Mean measured values on the $180 \mathrm{~cm}$ distance were $145 \pm 24,205 \pm 29,168 \pm 23$, and $172 \pm 21 \mathrm{~cm}$, respectively. The estimated lengths on the $180-\mathrm{cm}$ task were 18 out of 40 times between the lower and upper margins (45\%) and 16 times $(40 \%)$ outside the $15 \%$ margin. On the $210-\mathrm{cm}$ task, the surgeons measured $176 \pm 31,223 \pm 32,210 \pm 24$, and $221 \pm 24 \mathrm{~cm}$. The measurements were 18 out of 40 times between the $10 \%$ margins (45\%) and 13 times (33\%) outside the $15 \%$ margin. As shown in Table 2, the surgical residents measured a mean length of $137 \pm 31 \mathrm{~cm}$ on the $150-\mathrm{cm}$ task and $159 \pm 32 \mathrm{~cm}$ on the $180-\mathrm{cm}$ task. Mean measured length on the $210-\mathrm{cm}$ task was $183 \pm 40 \mathrm{~cm}$. In total, the residents estimated the lengths 25 out of 120 times between the $10 \%$ margin (21\%).

Deviation percentage of the goal length was compared between the surgeons and residents for each task separately (Table 3). The bariatric surgeons overestimated the $150-\mathrm{cm}$ and $210-\mathrm{cm}$ tasks with $4 \%(C I 0.4,9)$ and $1 \%$ $(C I-4,6)$, respectively. The $180-\mathrm{cm}$ goal length was underestimated by the surgeons with $6 \%(C I-11,-0.8)$. The surgical residents underestimated the lengths of the 150-, 180-, and 210-cm tasks with $12(C I-18,-6), 16$ $(C I-19,-13)$, and $18 \%(C I-22,-13)$, respectively. There was a significant difference between the surgeons and residents on all three measured tasks.

The individual deviation percentage and the interindividual difference between the surgeons are shown in Table 4 and Fig. 3. Bariatric surgeon 1 underestimated the lengths of all three task, with $12(C I-24,-0.4), 20$ $(C I-29,-10)$, and $16 \%(C I-26,-6)$, respectively. There were significant differences between surgeon 1 and the other three surgeons on all three measured tasks. On the 150-cm task, surgeon 2, 3, and 4 overestimated the length showing no difference between each other. On the 180$\mathrm{cm}$ goal length, an overestimation was seen in surgeon 2 , which significantly differed compared to the other surgeons. Surgeon 2, 3, and 4 showed no difference on the 210-cm task.

\section{Discussion}

This study shows that experienced bariatric surgeons estimate laparoscopic small bowel length with on average less than $10 \%$ deviation from the goal lengths. However, this still resulted in $51 \%$ of the estimated bowel lengths with more than $10 \%$ deviation and $30 \%$ with more than $15 \%$ deviation from the goal lengths. There were considerable inter-individual differences between the bariatric surgeons. Furthermore, surgical residents inaccurately estimated the small bowel length by structurally underestimating the limb lengths.

Acceptable variation in bowel length measurement was defined as less than $10 \%$ deviation from the goal limb length. Due to lack of literature, it is unknown which percentage deviation is still acceptable without causing clinical consequences in terms of weight loss and nutritional deficiencies. A deviation of $10 \%$ was used as acceptable cut-off value, because a higher deviation would result in an overlap of the different limb lengths used in bariatric surgery (Fig. 2). A more liberal margin still resulted in $30 \%$ of measurements outside $15 \%$ deviation. These percentages outside the margins can partially be explained by the inter-individual difference between the bariatric
Fig. 1 Laparoscopic set up. A Laparoscopic box trainer $\mathbf{B}$ small bowel of the porcine
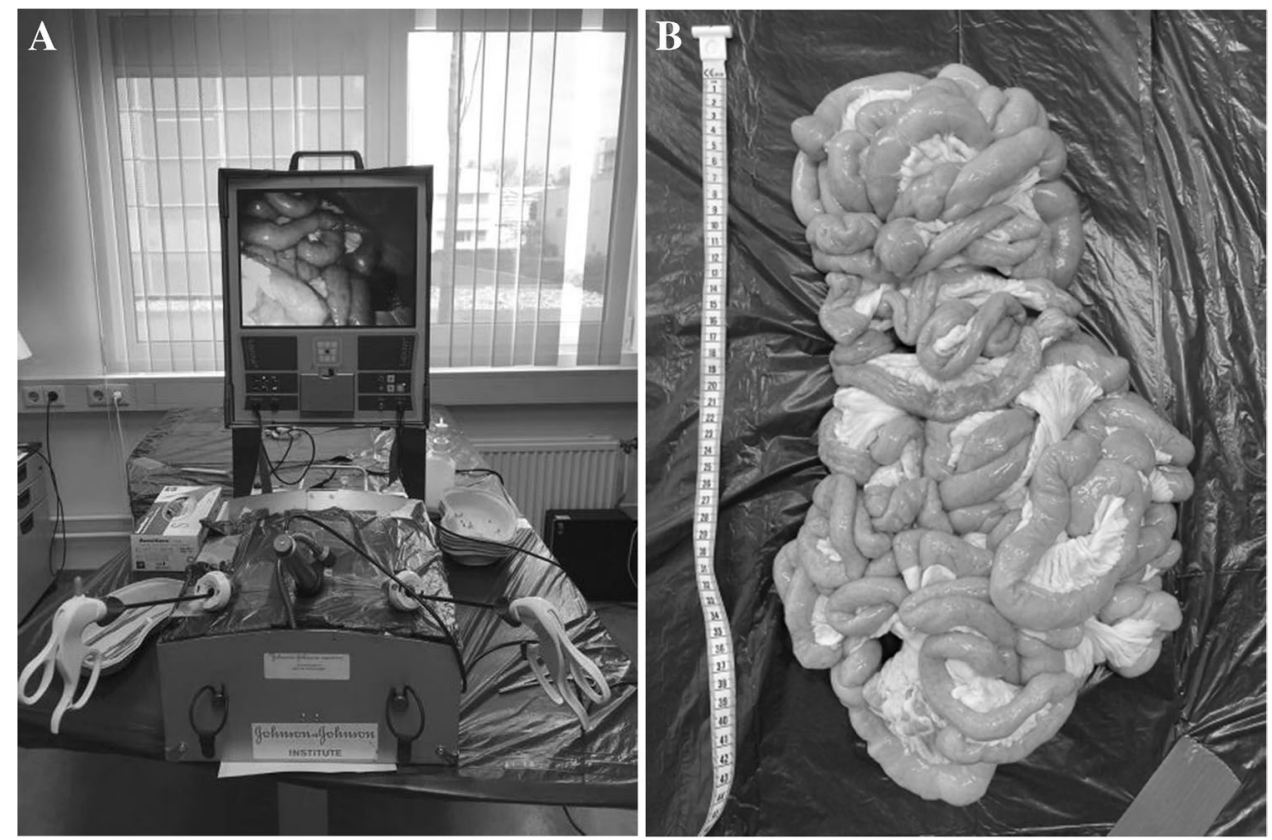
surgeons, as one of the bariatric surgeons structurally underestimated the small bowel length with more than $10 \%$ deviation. With regard to the structural underestimation, this is probably due to repeatedly estimating the steps too small. Nevertheless, the other three surgeons estimated

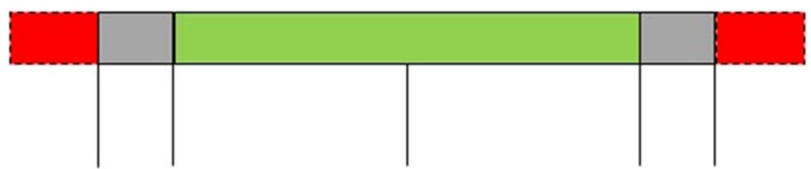

127,5135

150

165172,5

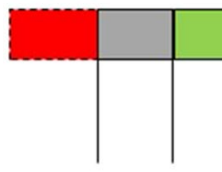

153172

180

198207

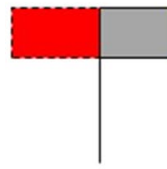

178,5189

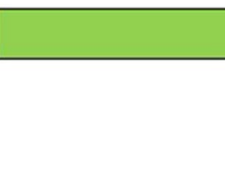

210

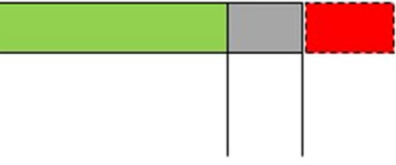

231241,5

Fig. 2 The acceptable cut-off value of $10 \%$ and the unacceptable cutoff point of $15 \%$ for the different measured limb lengths
$22 \%$ of the bowel segments with more than $15 \%$ deviation. With the hand-over-hand technique, the estimated steps can easily be affected by multiple factors such as the flexible structure of the small bowel with limited stretch due to the mesentery, two-dimensional imaging of a threedimensional bowel, counting error, or error of judgement, which all can result in outlying measurements. In daily practice, these deviations can result in bariatric patients with considerable difference between the goal and actual limb length. Nevertheless, the actual clinical consequences of these variations remain as yet unknown.

Comparable studies show deviations which are considerably higher compared to our results. The study of Gazer et al. assessed the reliability of laparoscopic bowel length measurement in 14 surgeons using an in vivo porcine model. They found that measured lengths were $36 \%$ shorter than the actual length, concluding that the assessment during laparoscopy was inaccurate [13]. However, the measurements were not performed by bariatric surgeons, which may explain the different outcomes, as general surgeons perform laparoscopic small bowel measurements to a much lesser extent than bariatric surgeons. Furthermore, they used non-marked laparoscopic graspers as a reference tool. A study of Isreb et al. investigated the effect of marking the graspers on measurement precision using a piece of string and a laparoscopic box trainer [11]. Greater accuracy

Table 1 Individual measured values of the bariatric surgeons

\begin{tabular}{|c|c|c|c|c|c|}
\hline & Bariatric surgeon 1 & Bariatric surgeon 2 & Bariatric surgeon 3 & Bariatric surgeon 4 & Total \\
\hline $150 \mathrm{~cm}, n$ & 10 & 9 & 10 & 10 & 39 \\
\hline Measured, cm & $131 \pm 25$ & $158 \pm 17$ & $156 \pm 19$ & $158 \pm 10$ & $151 \pm 21$ \\
\hline Deviation, $\mathrm{cm}$ & $-19 \pm 25$ & $8 \pm 17$ & $6 \pm 19$ & $8 \pm 10$ & $0.7 \pm 21$ \\
\hline Deviation, $\%$ & $-12 \pm 17$ & $5 \pm 11$ & $4 \pm 13$ & $5 \pm 7$ & $0.5 \pm 15$ \\
\hline Absolute deviation, $\%$ & $15 \pm 14$ & $9 \pm 8$ & $9 \pm 9$ & $7 \pm 5$ & $10 \pm 10$ \\
\hline Between margins $10 \%$ & 5 of 10 & 5 of 9 & 6 of 10 & 6 of 10 & 22 of 39 \\
\hline Outside $15 \%$ margins & 3 of 10 & 2 of 9 & 2 of 10 & 0 of 10 & 7 of 39 \\
\hline $180 \mathrm{~cm}, n$ & 10 & 10 & 10 & 10 & 40 \\
\hline Measured, cm & $145 \pm 24$ & $205 \pm 29$ & $168 \pm 23$ & $172 \pm 21$ & $172 \pm 32$ \\
\hline Deviation, $\mathrm{cm}$ & $-35 \pm 24$ & $25 \pm 29$ & $-12 \pm 23$ & $-8 \pm 21$ & $-8 \pm 32$ \\
\hline Deviation, $\%$ & $-20 \pm 13$ & $14 \pm 16$ & $-7 \pm 13$ & $-5 \pm 11$ & $-4 \pm 18$ \\
\hline Absolute deviation, $\%$ & $20 \pm 12$ & $17 \pm 12$ & $10 \pm 10$ & $10 \pm 7$ & $14 \pm 11$ \\
\hline Between margins $10 \%$ & 3 of 10 & 3 of 10 & 7 of 10 & 5 of 10 & 18 of 40 \\
\hline Outside $15 \%$ margins & 6 of 10 & 5 of 10 & 2 of 10 & 3 of 10 & 16 of 40 \\
\hline $210 \mathrm{~cm}, n$ & 10 & 10 & 10 & 10 & 40 \\
\hline Measured, cm & $176 \pm 31$ & $223 \pm 32$ & $210 \pm 24$ & $221 \pm 24$ & $208 \pm 33$ \\
\hline Deviation, $\mathrm{cm}$ & $-34 \pm 31$ & $13 \pm 32$ & $-0.3 \pm 24$ & $11 \pm 24$ & $-3 \pm 33$ \\
\hline Deviation, $\%$ & $-16 \pm 15$ & $6 \pm 15$ & $-0.2 \pm 11$ & $5 \pm 11$ & $-1 \pm 16$ \\
\hline Absolute deviation, $\%$ & $19 \pm 10$ & $13 \pm 9$ & $9 \pm 7$ & $10 \pm 7$ & $13 \pm 9$ \\
\hline Between margins $10 \%$ & 2 of 10 & 4 of 10 & 7 of 10 & 5 of 10 & 18 of 40 \\
\hline Outside $15 \%$ margins & 7 of 10 & 3 of 10 & 1 of 10 & 2 of 10 & 13 of 40 \\
\hline
\end{tabular}

Values are mean \pm standard deviation 
Table 2 Individual measured values of the residents

\begin{tabular}{llllll}
\hline & Resident 1 & Resident 2 & Resident 3 & Resident 4 & Total \\
\hline $150 \mathrm{~cm}, n$ & 10 & 10 & 10 & 10 & 40 \\
Measured, cm & $108 \pm 12$ & $172 \pm 28$ & $132 \pm 24$ & $138 \pm 18$ & $137 \pm 31$ \\
Deviation, cm & $-42 \pm 12$ & $22 \pm 28$ & $-18 \pm 24$ & $-12 \pm 18$ & $-13 \pm 31$ \\
Deviation, \% & $-28 \pm 8$ & $14 \pm 19$ & $-12 \pm 16$ & $-8 \pm 12$ & $-8 \pm 21$ \\
Absolute deviation, \% & $28 \pm 8$ & $18 \pm 14$ & $17 \pm 10$ & $12 \pm 8$ & $19 \pm 12$ \\
Between margins 10\% & 0 of 10 & 3 of 10 & 3 of 10 & 5 of 10 & 11 of 40 \\
Outside 15\% margins & 9 of 10 & 6 of 10 & 5 of 10 & 3 of 10 & 23 of 40 \\
180 cm, $n$ & 10 & 10 & 10 & 10 & 40 \\
Measured, cm & $130 \pm 15$ & $202 \pm 28$ & $149 \pm 9$ & $154 \pm 9$ & $159 \pm 32$ \\
Deviation, cm & $-50 \pm 15$ & $22 \pm 28$ & $-31 \pm 16$ & $-26 \pm 9$ & $-21 \pm 32$ \\
Deviation, \% & $-28 \pm 8$ & $12 \pm 16$ & $-17 \pm 9$ & $-14 \pm 5$ & $-12 \pm 18$ \\
Absolute deviation, \% & $28 \pm 8$ & $15 \pm 12$ & $18 \pm 9$ & $14 \pm 5$ & $19 \pm 10$ \\
Between margins 10\% & 0 of 10 & 3 of 10 & 1 of 10 & 2 of 10 & 6 of 40 \\
Outside 15\% margins & 10 of 10 & 4 of 10 & 6 of 10 & 4 of 10 & 24 of 40 \\
210 cm, $n$ & 10 & 10 & 10 & 10 & 40 \\
Measured, cm & $148 \pm 20$ & $229 \pm 28$ & $180 \pm 39$ & $175 \pm 18$ & $183 \pm 40$ \\
Deviation, cm & $-62 \pm 20$ & $19 \pm 28$ & $-30 \pm 39$ & $-35 \pm 18$ & $-27 \pm 40$ \\
Deviation, \% & $-29 \pm 9$ & $9 \pm 13$ & $-14 \pm 19$ & $-17 \pm 8$ & $-13 \pm 19$ \\
Absolute deviation, \% & $29 \pm 9$ & $12 \pm 10$ & $20 \pm 12$ & $17 \pm 8$ & $20 \pm 11$ \\
Between margins 10\% & 0 of 10 & 4 of 10 & 2 of 10 & 2 of 10 & 8 of 40 \\
Outside 15\% margins & 10 of 10 & 4 of 10 & 6 of 10 & 7 of 10 & 27 of 40 \\
\hline
\end{tabular}

Values are mean \pm standard deviation was found for the measurements performed with marked instruments. Furthermore, a study of Lusseden et al. evaluated the accuracy of stepwise laparoscopic small bowel measurement in residents and attendings using $500-\mathrm{cm}$ porcine intestine in a laparoscopic box trainer [14]. Both residents and attendings measured averaged $24 \mathrm{~cm}$ away from the $100-\mathrm{cm}$ goal, concluding there is a wide variability in both residents and attendings. They concluded that both groups should be educated to measure small bowel length more accurately. However, the study included no bariatric surgeons, and the participants performed several different step sizes to measure the bowel length. Another study compared the accuracy and precision of laparoscopic measurement with and without the aid of a measuring tool, using a laparoscopic box trainer and a rope [15]. The surgical residents underestimated with a mean of $128 \pm 42 \mathrm{~cm}$ on the $150-\mathrm{cm}$ goal length, comparable with the results of the residents in our experiment. Nevertheless, it is unknown if estimation with a rope is adequate with small bowel measurement, as it excludes relevant factors like the sensation and flexible structure of the intestinal tissue and the limited stretch of the mesentery.

Considering the inter-individual differences between the bariatric surgeons, all four surgeons had learned the handover-hand measurement technique in vivo from more experienced bariatric surgeons. Those experienced bariatric surgeons provided the surgeons feedback and decided whether they accurately estimated the limb lengths. Possible different learning methods, effects, and subjective assessments may have contributed to differences in results between the bariatric surgeons.

Table 3 Repeated measures analyses for the difference between surgeons and residents

\begin{tabular}{|c|c|c|c|c|}
\hline & \multirow[t]{2}{*}{ Estimate } & \multirow[t]{2}{*}{$P$-value } & \multicolumn{2}{|c|}{$95 \% C I$} \\
\hline & & & Lower & Upper \\
\hline \multicolumn{5}{|l|}{$150 \mathrm{~cm}, n=79$} \\
\hline Bariatric surgeons & 4 & 0.03 & 0.4 & 9 \\
\hline Residents & -12 & 0.0005 & -18 & -6 \\
\hline \multicolumn{5}{|l|}{ Differences } \\
\hline Surgeons vs residents & 16 & $<0.001$ & 9 & 23 \\
\hline \multicolumn{5}{|l|}{$180 \mathrm{~cm}, n=80$} \\
\hline Bariatric surgeons & -6 & 0.03 & -11 & -1 \\
\hline Residents & -16 & $<0.001$ & -19 & -13 \\
\hline \multicolumn{5}{|l|}{ Differences } \\
\hline Surgeons vs residents & 10 & 0.003 & 4 & 16 \\
\hline \multicolumn{5}{|l|}{$210 \mathrm{~cm}, n=80$} \\
\hline Bariatric surgeons & 1 & 0.56 & -4 & 6 \\
\hline Residents & -18 & $<0.001$ & -22 & -13 \\
\hline \multicolumn{5}{|l|}{ Differences } \\
\hline Surgeons vs residents & 19 & $<0.001$ & 12 & 26 \\
\hline
\end{tabular}

Dependent variable: percentage deviation (\%). Estimates of repeated measures analyses were determined for the group of bariatric surgeons and surgical residents. Estimates were compared to analyze the differences between bariatric surgeons and surgical residents. $C I$, confidence interval 
Table 4 Repeated measures analyses and inter-individual difference of the bariatric surgeons

\begin{tabular}{lllll}
\hline & Estimate & $P$-value & \multicolumn{2}{l}{$95 \%$ CI } \\
\cline { 4 - 5 } & & & Lower & Upper \\
\hline $150 \mathrm{~cm}, n=39$ & & & & \\
Bariatric surgeon 1 & -12 & 0.04 & -24 & -0.4 \\
Bariatric surgeon 2 & $5^{1}$ & 0.19 & -3 & 14 \\
Bariatric surgeon 3 & $4^{1}$ & 0.31 & -5 & 13 \\
Bariatric surgeon 4 & $5^{1}$ & 0.045 & 0.1 & 10 \\
180 cm, $n=40$ & & & & \\
Bariatric surgeon 1 & -20 & 0.001 & -29 & -10 \\
Bariatric surgeon 2 & $14^{1}$ & 0.03 & 2 & 25 \\
Bariatric surgeon 3 & $-7^{12}$ & 0.13 & -16 & 2 \\
Bariatric surgeon 4 & $-5^{12}$ & 0.23 & -13 & 4 \\
210 cm, $n=40$ & & & & \\
Bariatric surgeon 1 & -16 & 0.007 & -26 & -6 \\
Bariatric surgeon 2 & $6^{1}$ & 0.24 & -5 & 17 \\
Bariatric surgeon 3 & $-0.2^{1}$ & 0.97 & -8 & 8 \\
Bariatric surgeon 4 & $5^{1}$ & 0.18 & -3 & 13 \\
\hline
\end{tabular}

Dependent variable: percentage deviation (\%). Estimates of repeated measures analyses were determined for each bariatric surgeon individually. $C I$, confidence interval

${ }^{1}$ Significant difference compared to bariatric surgeon 1

${ }^{2}$ Significant difference compared to bariatric surgeon 2

\section{Individual percentage deviation of the surgeons}

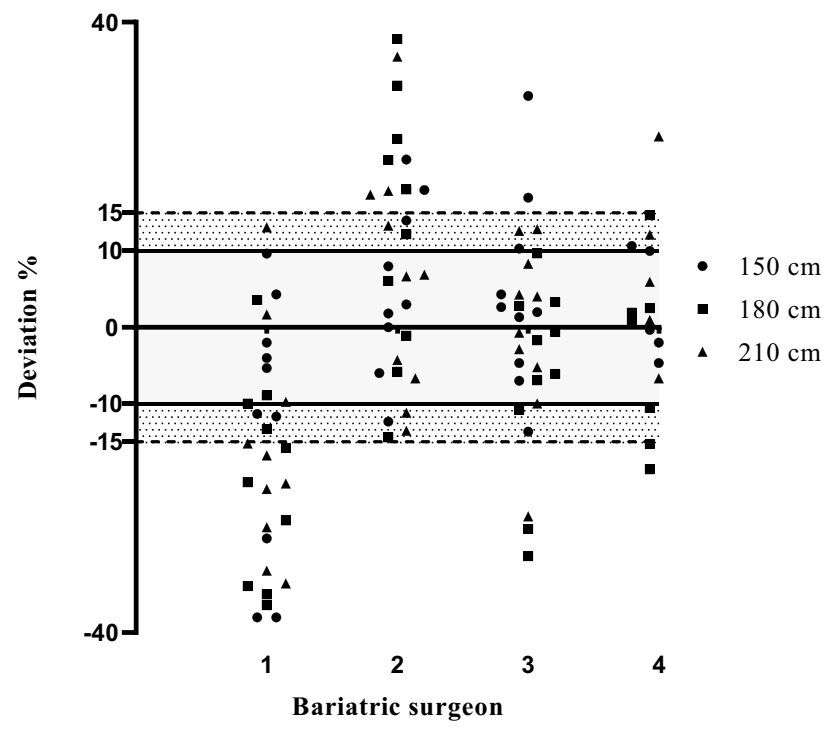

Fig. 3 Percentage deviation on all three tasks measured by the bariatric surgeons

To our knowledge, this is the first study investigating the intra- and the inter-individual variability of stepwise handover-hand laparoscopic bowel measurement in bariatric surgeons using a representative ex vivo model. As this stepwise measurement technique with marked graspers is used in a wide number of bariatric centers over the world, this is an important first step in evaluating this method in gastric bypass surgery [16]. In our bariatric center, this technique is performed in daily practice. Based on the results of the study, we continue using this measurement technique in gastric bypass surgery. The bariatric surgeon who structurally underestimated the lengths has adapted his measurement steps when performing the hand-over-hand measurement technique. As optimal limb length in bariatric surgery is a subject of many studies, differences between goal and actual limb length and differences among surgeons have possible consequences for studies investigating limb lengths. The results of this study emphasize that studies investigating limb lengths should incorporate results on the intra- and inter-individual variability of bowel length measurement in their center. It highlights the need for research to optimize this laparoscopic measurement technique and to evaluate different bowel length measurement techniques in bariatric surgery. Furthermore, the inaccurate estimation of the limb lengths by the surgical residents and the inter-individual differences between the bariatric surgeons both emphasize the need for a standardized learning method.

Limitations of this study were the use of an ex vivo model with devascularized porcine intestine, which caused a different sensation of the tissue and excluded the relevant factor of peristaltic bowel movements. The quality of the porcine intestine decreased during the day, which may have affected the measurements at the end of the day. An in vivo model would be preferable, but with the ex vivo model, it was possible to compare the estimated bowel lengths with the actual goal lengths, without having to perform animal experiments. Porcine intestine has several similarities with the human bowel, making it an appropriate model for this experiment [17]. The flexibility and sticky characteristics of the devascularized porcine intestine may have affected the tape measures determining the actual limb lengths; therefore, these measurements can also deviate from the actual limb lengths. Tape measures were performed twice to minimize this deviation. This study has a limited sample size with only four bariatric surgeons. However, this might be an adequate reflection of several bariatric centers, employing approximately four bariatric surgeons.

In conclusion, bariatric surgeons performed laparoscopic small bowel length measurements with an average less than $10 \%$ deviation from the goal lengths. However, this still resulted in $30 \%$ of the estimated lengths with more than $15 \%$ deviation. There were considerable inter-individual differences between the bariatric surgeons. Surgical residents structurally underestimated the limb lengths. Ascertainment of measurement accuracy and sufficient training of bariatric surgeons is essential for bariatric surgeries in which the limb length is of importance. 


\section{Appendix}

Tables 5 and 6

Table 5 Participant

demographics

\begin{tabular}{lllllll}
\hline & Sex & Age & $\begin{array}{l}\text { Years of experi- } \\
\text { ence/residency }\end{array}$ & Lap experience & $\begin{array}{l}\text { Bariatric } \\
\text { experience }\end{array}$ & $\begin{array}{l}\text { Lap bowel } \\
\text { measure- } \\
\text { ment }\end{array}$ \\
\hline Bariatric surgeon 1 & Male & 46 & 11 & $>3000$ & $>2000$ & $>1500$ \\
Bariatric surgeon 2 & Male & 43 & 11 & $>3000$ & $>2000$ & $>1500$ \\
Bariatric surgeon 3 & Female & 45 & 10 & $>3000$ & $>2000$ & $>1500$ \\
Bariatric surgeon 4 & Female & 36 & 5 & $>1000$ & $>500$ & $>500$ \\
Resident 1 & Male & 34 & 3 & $50-100$ & - & $10-20$ \\
Resident 2 & Male & 32 & 1 & $1-10$ & - & $1-10$ \\
Resident 3 & Male & 30 & 1 & $10-50$ & - & 0 \\
Resident 4 & Female & 32 & 2 & $1-10$ & - & $1-10$ \\
\hline
\end{tabular}

Lap experience, laparoscopic experience expressed as number of laparoscopic surgeries; lap bowel measurement, laparoscopic bowel measurement expressed as number of performed laparoscopic bowel measurements

Table 6 Inter-individual difference between bariatric surgeons

\begin{tabular}{|c|c|c|c|c|c|c|}
\hline & \multicolumn{2}{|c|}{ Bariatric surgeon 2} & \multicolumn{2}{|c|}{ Bariatric surgeon 3} & \multicolumn{2}{|c|}{ Bariatric surgeon 4} \\
\hline & Estimate & $P$-value & Estimate & $P$-value & Estimate & $P$-value \\
\hline \multicolumn{7}{|c|}{ Bariatric surgeon 1} \\
\hline $150 \mathrm{~cm}$ & -18 & 0.01 & -17 & 0.02 & -18 & 0.01 \\
\hline $180 \mathrm{~cm}$ & -33 & 0.0001 & -13 & 0.04 & -15 & 0.01 \\
\hline $210 \mathrm{~cm}$ & -22 & 0.004 & -16 & 0.02 & -21 & 0.002 \\
\hline \multicolumn{7}{|c|}{ Bariatric surgeon 2} \\
\hline $150 \mathrm{~cm}$ & & & 1 & 0.84 & 0.3 & 0.95 \\
\hline $180 \mathrm{~cm}$ & & & 20 & 0.006 & 18 & 0.01 \\
\hline $210 \mathrm{~cm}$ & & & 6 & 0.31 & 1 & 0.87 \\
\hline \multicolumn{7}{|c|}{ Bariatric surgeon 3} \\
\hline $150 \mathrm{~cm}$ & & & & & -1 & 0.85 \\
\hline $180 \mathrm{~cm}$ & & & & & -2 & 0.71 \\
\hline $210 \mathrm{~cm}$ & & & & & -5 & 0.31 \\
\hline
\end{tabular}

Dependent variable: percentage deviation (\%). Estimates of each bariatric surgeons individually were compared to analyze the inter-individual differences

\section{Declarations}

Ethics Approval For this type of study formal consent is not required.

Consent to Participate Informed consent was obtained from all individual participants included in the study.

Conflict of Interest The authors declare no competing interests.

Open Access This article is licensed under a Creative Commons Attribution 4.0 International License, which permits use, sharing, adaptation, distribution and reproduction in any medium or format, as long as you give appropriate credit to the original author(s) and the source, provide a link to the Creative Commons licence, and indicate if changes were made. The images or other third party material in this article are included in the article's Creative Commons licence, unless indicated otherwise in a credit line to the material. If material is not included in the article's Creative Commons licence and your intended use is not permitted by statutory regulation or exceeds the permitted use, you will need to obtain permission directly from the copyright holder. To view a copy of this licence, visit http://creativecommons.org/licenses/by/4.0/.

\section{References}

1. Robert M, Espalieu P, Pelascini E, Caiazzo R, Sterkers A, Khamphommala L, et al. Efficacy and safety of one anastomosis gastric bypass versus Roux-en-Y gastric bypass for obesity (YOMEGA): a multicentre, randomised, open-label, non-inferiority trial. Lancet. 2019;393:1299-309. 
2. Flum DR, Dellinger EP. Impact of gastric bypass operation on survival: a population-based analysis. J Am Coll Surg. 2004;199:543-51.

3. DeMaria EJ, Pate V, Warthen M, Winegar DA. Baseline data from American society for metabolic and bariatric surgery-designated bariatric surgery centers of excellence using the bariatric outcomes longitudinal database. Surg Obes Relat Dis [Internet]. Elsevier Inc.; 2010;6:347-55. Available from: http://dx.doi.org/https:// doi.org/10.1016/j.soard.2009.11.015

4. Mahawar KK, Parmar C, Carr WRJ, Jennings N, Schroeder N, Small PK. Impact of biliopancreatic limb length on severe proteincalorie malnutrition requiring revisional surgery after one anastomosis (mini) gastric bypass. J Minim Access Surg Medknow Publications. 2018; 14:37-43.

5. Ahuja A, Tantia O, Goyal G, Chaudhuri T, Khanna S, Poddar A. MGB-OAGB : effect of biliopancreatic limb length on nutritional deficiency, weight loss, and comorbidity resolution. Obesity Surgery; 2018;3439-45.

6. Zorrilla-Nunez LF, Campbell A, Giambartolomei G, Lo Menzo E, Szomstein S, Rosenthal RJ. The importance of the biliopancreatic limb length in gastric bypass: a systematic review. Surg Obes Relat Dis [Internet]. Elsevier Inc.; 2019;15:43-9. Available from: https://doi.org/10.1016/j.soard.2018.10.013

7. Choban PS, Flancbaum L. The effect of Roux limb lengths on outcome after Roux-en-Y gastric bypass: a prospective, randomized clinical trial. Obes Surg. 2002;12:540-5.

8. Pinheiro JS, Schiavon CA, Pereira PB, Correa JL, Noujaim P, Cohen R. Long-long limb Roux-en-Y gastric bypass is more efficacious in treatment of type 2 diabetes and lipid disorders in super-obese patients. Surg Obes Relat Dis. 2008;4:521-5.

9. Wagner M, Mayer BFB, Bodenstedt S, Kowalewski KF, Nickel F, Speidel S, et al. Comparison of Conventional methods for bowel length measurement in laparoscopic surgery to a novel computerassisted 3D measurement system. Obes Surg. 2021;

10. Inabnet WB, Quinn T, Gagner M, Urban M, Pomp A. Laparoscopic Roux-en-Y Gastric bypass in patients with BMI <50: a prospective randomized trial comparing short and long limb lengths. Obes Surg. 2005;51-7.
11. Isreb S, Hildreth A, Mahawar K, Balupuri S, Small P. Laparoscopic instruments marking improve length measurement precision. World J Laparosc Surg with DVD. 2009;2:57-60.

12. Sørensen SMD, Savran MM, Konge L, Bjerrum F. Three-dimensional versus two-dimensional vision in laparoscopy: a systematic review. Surg Endosc [Internet]. Springer US; 2016;30:1123. Available from: http://dx.doi.org/https://doi.org/10.1007/ s00464-015-4189-7

13. Gazer B, Rosin D, Bar-Zakai B, Willenz U, Doron O, Gutman $\mathrm{M}$, et al. Accuracy and inter-operator variability of small bowel length measurement at laparoscopy. Surg Endosc. Springer US; 2017;31:4697-704.

14. Ryan Lussenden, MD, David Brams, MD, Lee Sillin, MD, Dmitry Nepomnayshy MLC. Laparoscopic measurement of intestinal length: how accurate are we? (2012 Scientific Session of the Society of American Gas- trointestinal and Endoscopic Surgeons (SAGES) San Diego, California, USA, 7-10 March 2012 Poster Presentations) [Internet]. Surg Endosc. 2012 [cited 2021 May 25]. Available from: https://www.sages.org/meetings/annual-meeting/ abstracts-archive/laparoscopic-measurement-of-intestinal-lengthhow-accurate-are-we/

15. Jackson HT, Zettervall SL, Teitelbaum EN, Holzner M, Weissler $\mathrm{J}$, Amdur RL, et al. Effects of experience and reference tools on laparoscopic length measurements. Surg Endosc [Internet]. Springer US; 2015;29:1297-302. Available from: http://dx.doi. org/https://doi.org/10.1007/s00464-014-3802-5

16. Madan AK, Harper JL, Tichansky DS. Techniques of laparoscopic gastric bypass: on-line survey of American Society for Bariatric Surgery practicing surgeons. Surg Obes Relat Dis. 2008;4:166-72.

17. Kararli TT. Comparison of the gastrointestinal anatomy, physiolog, and biochemistry of humans and commonly used laboratory animals. Biopharm Drug Dispos. 1995;16:351-80.

Publisher's Note Springer Nature remains neutral with regard to jurisdictional claims in published maps and institutional affiliations.

\section{Authors and Affiliations}

\section{Nienke Slagter ${ }^{1,2}$ (1) . Mette van Wilsum ${ }^{1}$. Loek J. M. de Heide ${ }^{1}$. Ewoud H. Jutte ${ }^{1}$ - Mirjam A. Kaijser ${ }^{1}$. Stefan L. Damen ${ }^{1}$. André P. van Beek ${ }^{3}$. Marloes Emous ${ }^{1}$}

\author{
Mette van Wilsum \\ Mette.van.Wilsum@mcl.nl \\ Loek J. M. de Heide \\ L.de.Heide@mcl.nl \\ Ewoud H. Jutte \\ Ewoud.Jutte@mcl.nl \\ Mirjam A. Kaijser \\ Mirjam.Kaijser@mcl.nl \\ Stefan L. Damen \\ Stefan.Damen@mcl.nl \\ André P. van Beek \\ A.P.van.Beek@umcg.nl \\ Marloes Emous \\ Marloes.Emous@mcl.nl
}

1 Center for Obesity Northern Netherlands (CON), Medical Center Leeuwarden, Henri Dunantweg 2, 8934 AD Leeuwarden, the Netherlands

2 University Medical Center Groningen, University of Groningen, Hanzeplein 1, 9713 GZ Groningen, the Netherlands

3 Department of Endocrinology, University of Groningen, University Medical Center Groningen, Hanzeplein 1, 9713 GZ Groningen, the Netherlands 